\title{
Annotations
}

\section{Does it matter if atrial septal defects are not diagnosed in childhood?}

Does it matter if atrial septal defects are not diagnosed in childhood? It is only important to diagnose atrial septal defect (ASD) in childhood if the defect carries an adverse prognosis that can safely and effectively be improved by treatment. As this defect is largely asymptomatic during childhood, it is necessary also to know whether delayed diagnosis and therefore treatment adds to the risks or prejudices the long term result.

The three types of ASD are the ostium secundum, which is by far the most common and is a defect in the region of the fossa ovalis, sinus venosus ASD sited at the junction of the superior vena cave and usually associated with anomalous drainage of the right upper lobe pulmonary veins, and ostium primum defects. Ostium secundum and sinus venosus ASDs behave similarly and are usually isolated. Ostium primum ASDs, more properly called partial atrioventricular canal or endocardial cushion defects, are sited inferiorly, are frequently complicated, and are usually diagnosed and surgically treated in infancy or childhood. They are not the subject of this annotation.

Isolated ASD is rarely diagnosed in the neonate because shunting is established only gradually. The left to right shunt is dependent on the greater distensibility of the right heart chambers and lower resistance of the pulmonary compared with the systemic circuit. Pulmonary blood flow gradually increases as the right ventricle thins after fall of the high fetal pulmonary vascular resistance but pulmonary artery pressure remains normal. In the majority of cases the defect is large, does not restrict flow, and there is only a potential gradient between the low pressure left and right atria. In a minority the defect remains small and restrictive. Because there is no communication between the high pressure left ventricle or aorta and the pulmonary circuit, pulmonary hypertension is never 'obligatory' as in non-restrictive ventricular septal defect and the pulmonary artery pressure falls normally after birth.

\section{Prevalence}

ASD is said to account for $10 \%$ of congenital heart disease at birth and for about $25 \%$ of adult patients with congenital heart disease in whom it is the commonest congenital cardiac defect after bicuspid aortic valve. It is two or three times more common in women and although more and more ASDs are being diagnosed and closed during childhood, many are still only first identified in adult life or even in old age. This is because the clinical features of ASD in the adult can be subtle. The diagnosis is easier in children. Most children, but not all adults, have obvious signs and any child with a murmur is usually referred for echocardiography. In adults both symptoms and obvious murmurs may be lacking and the diagnosis may never be made.

\begin{abstract}
Diagnosis
Most children have a pulmonary systolic murmur, fixed splitting of the second heart sound, and inspiratory mid-diastolic tricuspid flow murmur. The electrocardiogram shows sinus rhythm with normal or rightward frontal QRS axis and partial right bundle branch block. This may be undramatic but should always raise the possibility of ASD. Left axis deviation suggests a partial atrioventricular canal (ostium primum) defect. The chest radiograph may show a barely enlarged heart but there is usually slight prominence of the right atrium and main pulmonary artery and suggestion of pulmonary plethora. Echocardiography is diagnostic and permits calculation of the right ventricular and pulmonary artery pressures and the size of the shunt as well as direct visualisation of the defect and pulmonary venous connections. Cardiac catheterisation is unnecessary in children.
\end{abstract}

\section{Natural history}

Textbooks of cardiology continue to teach that in the absence of pulmonary vascular disease all ASDs should be closed, ${ }^{1}$ or that closure should be advised for all patients with uncomplicated ASDs in whom there is evidence of significant shunting, that is pulmonary to systemic flow ratio (Qp:Qs) exceeding about 1.5:1. ${ }^{2}$ This advice is based on early perception of the natural history of the untreated defect in the presurgical era and before echocardiography had begun to reveal a whole new population of ASDs, which had formerly not been diagnosed at all. The patients described in the early studies of the natural history of ASD were recognised because they had the florid clinical and radiological evidence of heart disease that were then needed to justify cardiac catheterisation. They represented only the worst end of the ASD spectrum. Both children and adults with uncomplicated and asymptomatic ASD were regarded, even if seen by a cardiologist, as having mild pulmonary valve stenosis, 'idiopathic dilatation of the pulmonary artery', or innocent murmurs.

ASD was thought to be associated with progressive morbidity and greatly reduced survival. These opinions, which are still quoted, ${ }^{13}$ were not based on long follow up because ASD had only slowly begun to be recognised clinically after papers by Bedford et al in $1941^{4}$ and by Wood after his St Cyres lectures in $1950 .^{5}$ The fixed splitting of the second heart sound was only published in $1956 .^{6}$ Lacking personal follow up of only recently diagnosed patients, cardiologists compared the prevalence of symptoms and complications in the different age groups and came to the conclusion that ASD had a relatively poor prognosis. Based on this apparently dismal outlook the earliest open heart surgery was practised on patients with ASD whose defects could be closed quickly at a time when speed was all important under conditions of hypothermic cardiac arrest. Surgical repair on diagnosis became the rule 
in both children and adults. Because this practice has been conscientiously followed since the late $1950 \mathrm{~s}$, all that is known of the natural history of the untreated defect is based on that early glimpse of a fragment of the ASD population.

The early writers believed that patients with ASDs were likely to have a poor outcome. For example, Campbell et al calculated an annual rate of attrition of $4.5 \%$ for the fourth decade, $5.4 \%$ for the fifth, and $7.5 \%$ for the sixth. ${ }^{78}$ That meant that the average patient with ASD would not be expected to live beyond the age of 50 . Using the same methods, Craig and Seltzer found that three quarters of their patients with ASD who were between the ages of 18 and 56 were symptomatic. Pulmonary hypertension was the commonest adverse predictive factor (although only $15 \%$ of their patients had a pulmonary vascular resistance of over 5 Wood units). ${ }^{9}$ Markman et al attributed deterioration to the development of atrial fibrillation, recurrent bronchitis, pulmonary hypertension, and pulmonary infarction. They observed that the development of heart failure was often associated with the onset of atrial fibrillation. Only $7 \%$ of patients in the third decade compared with $30 \%$ in the fifth and sixth decades had a pulmonary artery systolic pressure of over $50 \mathrm{~mm} \mathrm{Hg}$ and $60 \%$ of their patients aged 40 or over were in New York Health Association (NYHA) classes III and IV, $30 \%$ by the age of 50 or over and $49 \%$ by 60 years. ${ }^{10}$ The pulmonary artery mean pressure was raised in only $14 \%$ of patients under 20 years compared with $53 \%$ of patients over 40 years in the series of Hamilton et al. ${ }^{11}$

Dyspnoea, fatigue, and palpitations are the most common symptoms. Only $8 \%$ of children aged 1 to 10 years in the study of Hamilton et al were symptomatic compared with $35 \%$ of patients aged over 40 years. The progressive increase in prevalence of symptomatic limitation as age advances has been emphasised in most studies. Markman et al regarded pulmonary infection as a factor predicting clinical deterioration, while Adams blamed pulmonary infection for the development of pulmonary hypertension. ${ }^{12}$ Unlike atrial arrhythmias, the development of pulmonary hypertension has not been related to shunt size and it does not seem that a raised pulmonary blood flow is the cause as this is not more than the cardiac output on modest exercise in a normal person. Unlike other writers, Craig and Seltzer concluded that younger adults are more prone to develop this complication. They had probably observed patients with anatomical ASDs and Eisenmenger's syndrome who are frequently young, with small hearts, and no evidence that they have ever developed a high pulmonary blood flow. Wood's theory was that there had been congenital persistence of the high fetal pulmonary vascular resistance after birth. Whatever the reason for the association of a primary type pulmonary hypertension with an anatomical ASD, the number of such patients included in the reports on the natural history of ASD will strongly bias the figures. These young patients are quite different from the $5 \%$ of older patients with ASD whose raised pulmonary vascular disease develops later in life usually in association with atrial arrhythmia and progressive tricuspid regurgitation. This tricuspid regurgitant volume usurps shunted volume and right ventricular forward flow diminishes. The reduced flow velocity in the dilated pulmonary arteries leads to laminar thrombosis and repeated peripheral platelet emboli with gradually rising pressure. This accounts for the observations of Markman et al of pulmonary infarction as a feature of deterioration in his pulmonary hypertensive older patients. In patients with severe disability pulmonary hypertension is an important contributor. It is associated with clinical deterioration and poor life expectancy and was a feature in
$82 \%$ of the patients of Gault et al who were in NYHA classes III and IV compared with only $24 \%$ of those in classes I and II. ${ }^{13}$

Although children and young adults with ASD may have considerable athletic ability and endurance, it has long been felt that left ventricular dysfunction contributes to the development of symptomatology. ${ }^{14}$ Gault et al observed that major right to left shunting is rare even in patients with pulmonary hypertension and suggested that this is because of increases in left ventricular diastolic stiffness with age or that the development of left ventricular systolic dysfunction might act to maintain left to right shunting. Right ventricular dilatation deforms the geometry of the left ventricle reducing its size, impairing diastolic compliance, and diminishing left ventricular volume reserve, which is also diminished by inability to invoke the Starling mechanism. This may activate the renin-angiotensin system but an increased left ventricular end diastolic pressure cannot be given as evidence of left ventricular dysfunction because in diastole all four cardiac chambers are in open communication and their pressures are similar. Recent studies have shown normal left ventricular function after closure of ASD and that preoperative limitation of stroke volume is due to reversible mechanical factors. ${ }^{15-17}$

Small right to left shunts can be demonstrated even in uncomplicated ASDs and transient right to left shunting with paradoxical embolism has been blamed for otherwise unexplained stroke in healthy adults with a patent foramen ovale ${ }^{18}$ or atrial septal aneurysm. ${ }^{19}$

The onset of atrial flutter or less commonly fibrillation, nearly always marks a step down in the course of patients with ASD and the incidence increases with age, being found in half of the surgical series of Sutton et al whose patients were aged 60 years or over. ${ }^{20}$ Craig and Seltzer found it to be a more important adverse prognostic feature even than pulmonary hypertension. Atrial fibrillation is most likely to develop in patients with both volume and pressure overloaded right ventricles. Its onset brings a risk of both pulmonary and systemic embolism and these complications would explain Campbell's observation that most patients died within 5 or 10 years of its onset. Some patients with ASD achieve considerable longevity even into their $90 \mathrm{~s} .{ }^{21}$ It would be helpful to recognise patients with ASD programmed to reach old age without major disability despite this malformation.

The value of surgical intervention in ASD has been exhorted after comparison of the subsequent progress of patients after ASD closure with that of historic controls with ASD described from a presurgery, preechocardiographic era. ${ }^{20}$ These were skewed populations and such comparison is clearly invalid. More realistically Murphy et al studied 123 patients whose defects had been closed between 27 and 32 years earlier at the Mayo Clinic and compared them with age matched normal controls. ${ }^{22}$ The actuarial survival rate among young patients aged 11 to 24 years at the time of surgery was found to be similar to the controls but the survival rate in patients whose defects were closed when they were over age 25 years was less good. The survival of patients operated on when over age 41 was considerably worse, $40 \%$ compared with $59 \%$ in the controls. The excess mortality was due to an unchanged risk of onset of arrhythmias and continued throughout the follow up period due to the association with stroke and heart failure. The administration of anticoagulants to patients who have started to develop atrial arrhythmias is obviously very important.

People with ASD may remain asymptomatic or minimally symptomatic and undiagnosed until middle or later age. At Hammersmith Hospital routine surgical closure has not been the practice in such patients. A comparison of 
the long term health of medically compared with surgically treated patients showed no difference between 34 patients managed medically and 48 who had undergone surgical repair. ${ }^{23}$ The two groups were similar with respect to age, functional class, and haemodynamic characteristics at the time of entry which was before the echocardiographic era. There were no operative or perioperative deaths and after a mean follow up of 25 years overall survival was more than $90 \%$ in both groups whose mean age was 63 years in each. Symptomatic status, prevalence of atrial fibrillation, and use of diuretics was also similar. Clearly the prognosis of the medically treated patients was much better than that of the historical controls described previously, although it was not normal but then nor was that of the group who had undergone late surgical closure. This study was quite different from that of Konstantinides et al who compared the long term course of 179 patients with isolated ASD diagnosed after the age of 40 years, 84 of whom had their defects closed and 95 were followed up medically. ${ }^{24}$ Twenty per cent of the patients had initially been followed up medically for up to 16 years before a decision was made to operate on them. The mean follow up ranged from one to 26 years (mean 8.9 years). The surgical mortality was $2.8 \%$. Surgical repair was found to increase survival and limit functional deterioration but this favourable effect of surgical treatment was only prominent in the subgroup of patients with severe preoperative heart failure (NYHA classes III or IV) among whom $69 \%$ had long term improvement. This is not in contention. Such patients had not been included in the Hammersmith study of largely asymptomatic ASDs.

Recent studies have revealed a new population of patients with ASD discovered through the growing application of echocardiography. Sixty nine patients aged 70 years or older were described from the Mayo Clinic with ages ranging between 70 and 93 years. ${ }^{25}$ Forty per cent of the patients had not been suspected of having an ASD before the echo study and over $40 \%$ were in NYHA class I. Interestingly the strongest overall predictors of survival included atrial fibrillation. The others were functional class, left ventricular ejection fraction, and pulmonary artery systolic pressure. This observation makes sense. Although the onset of atrial arrhythmia often initiates tricuspid regurgitation, the development of congestive features and possibly pulmonary vascular disease, patients with atrial fibrillation will continue to do well provided the onset of the arrhythmia has not been marked by the development of these features. An analysis of 289 cases of ASD aged 50 or over from Tokyo showed that nearly a third were asymptomatic and a fifth had normal or nearly normal pulmonary artery pressures. ${ }^{26} \mathrm{~A}$ third of these patients had shunt ratios of less than $2: 1$ and the authors concluded that conservative management may be appropriate in asymptomatic older patients with normal pulmonary artery pressures and that ASD can be compatible with a normal life span.

\section{Does it matter if ASDs are not diagnosed in childhood?}

Closure of ASD in children and young adults recreates a dependably normal or near normal prognosis with very low mortality and morbidity (never 'negligible' or 'acceptable') for the price of a scar on the chest and in the knowledge that a number of defects will have been closed unnecessarily.

The development of device closure by a pair of occluding discs may permit non-operative closure of smaller well defined defects but these are probably the sort that can safely be left alone. It is likely that smaller restrictive defects with smaller shunts carry a better and even normal prognosis. There is no doubt that the more florid defects defined by heart size, (specifically right atrial and right ventricular volumes), defect and shunt size, should be closed during childhood. Lesser defects need to be fully documented by defect size, chamber volume and shunt size and then carefully followed so that the future need for their closure either by device or surgically can be ascertained for future generations. A few adults may have more late morbidity with or without later closure than they would have had if their defects had been closed earlier.

The natural history of ASD is still known only in part. ${ }^{27}$ The defect does not invariably reduce health and activity or limit prognosis and routine closure is not of proven benefit. ${ }^{28}$ Development of atrial arrhythmias is common in middle or later years and leads to increased morbidity but the threat of thromboembolism can be reduced by use of anticoagulants. The risk of late development of pulmonary vascular disease is small but is a definite indication for defect closure before it advances further.

Yes, it is better to diagnose ASD in childhood than to miss it but there is no indication for closure of asymptomatic ASDs first diagnosed in middle or old age.

Department of Cardiology

CELIA M OAKLEY

Hammersmith Hospital,

Du Cane Road

London W12 ONN

1 Tynan M, Anderson RH. Congenital heart disease. In: Julian D, Camm J Fox K, et al. Disease of the heart. 2nd Ed. London: Saunders, 1996: Fox

2 Friedman WF. Congenital heart disease in infancy and childhood. In: Braunwald $\mathrm{E}$, ed. Heart disease. $A$ textbook of cardiovascular medicine. 4th Braunwald E, ed. Heart disease. A textbo

3 Perloff JK. Congenital heart disease in adults. In: Braunwald E, ed. Heart disease. $A$ textbook of cardiovascular medicine. 4th Ed. Philadelphia: Saunders, 1992: 968.

4 Bedford DE, Papp C, Parkinson J. Atrial septal defect. Br Heart $\mathcal{f} 1941$; 3: 37-68.

5 Wood P. Congenital heart disease (St Cyres lectures). BMF 1950; ii: 639 and 693.

6 Leatham A, Gray I. Auscultatory and phonocardiographic signs of atrial septal defect. Br Heart $\mathcal{F}$ 1956; 18: 193-6.

7 Campbell M, Neill C, Suzman S. The prognosis of atrial septal defect. BMF 1957 ; i: $1375-83$.

8 Campbell M. Natural history of atrial septal defect. Br Heart $\mathcal{f} 1970$; 32: 820-6.

9 Craig RJ, Selzer A. Natural history and prognosis of atrial septal defect. Circulation 1968; 37: 805-15.

10 Markman P, Howitt G, Wade EG. Atrial septal defect in the middle-aged and elderly. $Q f$ Med $1965 ; 34: 409-26$.

11 Hamilton WT, Haffajee CI, Dalen JE, Dexter L, Nadas AS. Atrial septal defect secundum type: clinical profile with physiologic correlates in defect secundum type: clinical profile with physiologic correlates in children and adults. In: Roberts W

12 Adams C. A reappraisal of life expectancy with atrial septal defect of the secundum type. Diseases of the Chest 1965; 48: 357-75.

13 Gault JH, Morrow AG, Gay WA, Ross J. Arial septal defect over the age of forty years: clinical and hemodynamic studies and the effects of operation. Circulation 1968; 37: 261-72.

14 Dexter L. Atrial septal defect. Br Heart f 1956; 18: 209-25.

15 Bonow RO, Borer JS, Rosing DR, Bacharach SL, Green MV, Kent KM. Left ventricular functional reserve in adult patients with atrial septal defect: pre- and post-operative studies. Circulation 1981; 63: 1315-22.

16 Carabello BA, Gash A, Mayers D, SpannJF. Normal left ventricular systolic function in adults with atrial septal defect and left heart failure. Am $\mathcal{f}$ Carfunction in adults with at

17 Booth KM, Wisenbaugh T, Smith M, De Maria AN. Left ventricular distensibility and passive elastic stiffness in atrial septal defect. $₹$ Am Coll Cardio 1988; 12: 1231-6.

18 Lynch J, Schuchard GH, Gross CM, Wann L. Prevalence of right to left atrial shunting in a healthy population: detection by valsalva manoeuvre contrast echocardiography. Am $\mathcal{F}$ Cardiol 1984; 53: 1478-80.

19 Marazanof M, Roudaut R, Cohen A, et al. Atrial septal aneurysm. Morphological characteristics in a large population: pathological associations. A French multicenter study on 259 patients investigated by transoesophageal echocardiography. Int $\mathcal{A}$ Cardiol 1995; 52: 59-65.

20 St John Sutton MG, Tajik AG, McGoon DC. A septal defect in patients aged 60 years or older: operative results and long-term follow-up. Circulation $1981 ; 64: 402-9$.

21 Perloff JK. Ostium secundum atrial septal defect-survival for 87 and 94 years. Am $\mathcal{f}$ Cardiol 1984; 53: 388-9.

22 Murphy JG, Gersh BJ, McGoon MD, et al. Long-term outcome after surgical repair of isolated atrial septal defect. $N$ Engl $f$ Med 1990; 323: 1645-50. 
23 Shah D, Azhar M, Oakley CM, Cleland JGF, Nihoyannopoulos P. Natural history of secundum atrial septal defect in adults after medical or surgical treatment: a historical prospective study. Br Heart $\mathcal{F}$ 1994; 71: 224-8.

24 Konstantinides S, Geibel A, Olschewski M, et al. A comparison of surgical and medical therapy for atrial septal defect in adults. N Engl f Med 1995; 333: 469-73.

25 Foster-Smith KW, Murphy JG, Bailey KR, et al. Secundum atrial septal defect (ASD): clinical profile and surgical experience in septuagenarians and older. Proceedings of the American College of Cardiology Meeting. Atlanta, Georgia, 1994; 465A: 826-32.
26 Ueda K. Clinical profiles, pathologic spectrum and management of atrial septal defect in patients aged 50 or over: how should they be treated? $f$ Cardiogr 1984; 14: 129-36.

27 Konstantinides S, Geibel A, Kasper W, et al. The natural course of atrial septal defect in adults-a still unsettled issue. Klin Wochenschr 1991; 69: 506-10.

28 Ward C. Secundum atrial septal defect: routine surgical treatment is not of proven benefit. Br Heart $¥$ 1994; 71 : 219-23.

\section{Cystic fibrosis in teenagers and young adults}

The millennium will see over 6000 patients with cystic fibrosis in the UK, half of whom will be teenagers and adults, the over 15 age group increasing by about 120 per year. ${ }^{1}$ We are not providing sufficient resources for this latter group now, nor are we making sufficient commitment to their future needs. There are three reasons for this. Firstly, some fault rests with the apparent failure of the NHS hospital trusts' management to deploy income derived from purchaser provision for cystic fibrosis care back into the cystic fibrosis service. There is also their persistent failure to meet the staffing levels recommended for cystic fibrosis centres by the British Paediatric Association (BPA), the British Thoracic Society, the Cystic Fibrosis Trust, ${ }^{2}$ and the Royal College of Physicians ${ }^{3}$ and their alleged failure to have established a realistic cost for treatments. Secondly, there are a significant minority of professional colleagues who refuse to refer older patients with this multisystem disease to cystic fibrosis centres with appropriate multidisciplinary teams. Finally, some fault also rests with those of us who have a major commitment to cystic fibrosis care failing to initiate a proper training programme with recognised accreditation for junior doctors wishing to care for patients with cystic fibrosis.

\section{Special needs of the teenage patient with cystic} fibrosis: the 'transitional' clinic

Inevitably close ties develop between the paediatric medical and nursing teams and the patients and their families. Important though these are in giving the child and family emotional security, they must be loosened and eventually broken to allow the patient to grow into independence, and realise responsibility for self. Teenagers with cystic fibrosis can only grow into adulthood in an environment designed to cultivate their independence. Close collaboration between paediatric and adult cystic fibrosis teams is essential so that a system of care is established within which the patient's transition through the turbulence of adolescence is as smooth as possible. In Leeds we believe that this is best achieved in a flexible 'transitional' clinic, run by staff from both the paediatric and adult teams. Patients usually transfer from paediatric to transitional clinic at about 14 years of age, and from transitional to adult clinic, according to individual needs, between 16 and 18 years of age. A survey of our own patients showed that knowledge of the transitional clinic had reduced patients' anxieties about leaving the paediatric unit, and getting to know the adult carers, before moving on to the adult clinic proper, had reassured them. ${ }^{4}$

\section{Cystic fibrosis centres for young adults}

The teenager with cystic fibrosis must be allowed to leave the paediatric unit and the paediatrician behind. There is no justification for the paediatrician retaining the reins of care either because of the spurious excuse that no adult respiratory specialist will give the level of commitment required, or to maintain 'patient numbers' in the NHS market economy. Nor should 'adult' clinics run by a paediatrician and chest physician be seen as fulfilling a need outside of the transitional clinic referred to above. The paediatrician has to 'let go'. But responsibility for continuing care must involve an adult cystic fibrosis centre. If, as paediatricians, we accept the argument for centred care for children with cystic fibrosis, then similarly we must accept centred care for adult patients.

Some with responsibility for older patients with cystic fibrosis believe that all of them should receive total care at the cystic fibrosis centre. Why? Because cystic fibrosis is a multisystem, complex disease and, with present high standards of care in paediatric centres, multisystem complications are often confined to adults. Hence, not only must a critical mass of experience be learned so that one is alerted to complications early, but colleagues in many disciplines (for example obstetrics and gynaecology, endocrinology, rheumatology, gastroenterology, surgery), must become familiar with the problems presented in their own fields by adult patients with cystic fibrosis. A sufficient patient 'through-put' can take place only in a cystic fibrosis centre. Access to specialist paramedical personnel and to basic, not esoteric, investigations is significantly easier within specialist clinics. ${ }^{5}$ Patients cared for by cystic fibrosis specialist staff receive more intensive treatment, especially with regard to nutrition, and are significantly more likely to adhere to physiotherapy regimens and to be self reliant. Physicians who believe that they can offer optimal care by looking after a few patients with a physiotherapist, dietitian, and social worker unschooled in the special needs of cystic fibrosis, are wrong. Units which believe that they can offer a safe and optimal home based intravenous antibiotic treatment programme without a cystic fibrosis liaison nurse are wrong. Young adult patients have voiced clearly their concerns about the fall in quality service they have experienced on transfer from specialist paediatric cystic fibrosis units to general adult chest clinics. ${ }^{5}$

There has been a marked increase over the last five years in the numbers of older patients attending specialist clinics. Seventy seven per cent now receive full or shared care from a cystic fibrosis centre. Ninety eight per cent want some input to their care from a specialist centre and $96 \%$ of patients not currently receiving centre care would prefer it if they were. ${ }^{5}$ Existing centres must be properly resourced to cope with these rapidly increasing numbers, and new centres set up in areas of need.

\section{What an adult centre must offer}

First and foremost the centre must be adequately staffed and have access to adequate bed numbers, preferably on a dedicated cystic fibrosis ward. It is unlikely that anyone other than a full time cystic fibrosis physician will be able to care properly for more than 100 patients. Therefore most large units will require two consultants. Teenagers and adults should have easy access to significant amounts of their doctor's time. Issues such as career choices, life 